\title{
Palmitoylated PrRP analog decreases body weight in DIO rats but not in ZDF rats
}

\author{
Martina Holubová1, Jana Zemenová1,2, Barbora Mikulášková1,3, \\ Vladimíra Panajotova4, Jiří Stöhr4, Martin Haluzík5, Jaroslav Kuneš1,3, \\ Blanka Železná1 and Lenka Maletínská1 \\ IInstitute of Organic Chemistry and Biochemistry, Academy of Sciences of the Czech Republic, \\ Prague, Czech Republic \\ 2Department of Analytical Chemistry, University of Chemistry and Technology, Prague, Czech Republic \\ IInstitute of Physiology, Academy of Sciences of the Czech Republic, Prague, Czech Republic \\ ${ }^{4}$ Apigenex Limited, Prague, Czech Republic \\ ${ }^{5}$ First Faculty of Medicine, Charles University, Prague, Czech Republic
}

Correspondence should be addressed to L Maletínská

Email

maletin@uochb.cas.cz

\begin{abstract}
Anorexigenic neuropeptides produced and acting in the brain have the potential to decrease food intake and ameliorate obesity, but are ineffective after peripheral application, owing to a limited ability to cross the blood-brain barrier. We have designed lipidized analogs of prolactin-releasing peptide ( $\operatorname{PrRP})$, which is involved in energy balance regulation as demonstrated by obesity phenotypes of both Prrp-knockout and Prrp receptor-knockout mice. The aim of this study was to characterize the subchronic effect of a palmitoylated PrRP analog in two rat models of obesity and diabetes: diet-induced obese Sprague-Dawley rats and leptin receptordeficient Zucker diabetic (ZDF) rats. In the rats with diet-induced obesity (DIO), a two-week intraperitoneal treatment with palmitoylated PrRP lowered food intake by $24 \%$ and body weight by $8 \%$. This treatment also improved glucose tolerance and tended to decrease leptin levels and adipose tissue masses in a dose-dependent manner. In contrast, in ZDF rats, the same treatment with palmitoylated PrRP lowered food intake but did not significantly affect body weight or glucose tolerance, probably in consequence of severe leptin resistance due to a nonfunctional leptin receptor. Our data indicate a good efficacy of lipidized PrRP in DIO rats. Thus, the strong anorexigenic, body weight-reducing, and glucose tolerance-improving effects make palmitoylated PrRP an attractive candidate for anti-obesity treatment.
\end{abstract}

Journal of Endocrinology (2016) 229, 85-96

\section{Introduction}

Obesity is a frequent metabolic disorder with a steadily increasing prevalence worldwide. Despite tremendous efforts, there is still a lack of weight-lowering pharmacotherapies that would be both efficacious and

\section{Key Words}

- prolactin-releasing peptide

- lipidization

- diet-induced obesity

- ZDF rats

food intake

- rat safe for the long-term (Yanovski \& Yanovski 2014). It is accepted that an enormous rise in the prevalence of obesity around the world is primarily the result of increased caloric intake and decreased physical activity. 
As obesity triggers other life-threatening diseases, including type 2 diabetes mellitus, hypertension, dyslipidemia, and atherosclerosis (Simmons et al. 2010, Vaneckova et al. 2014), an effective anti-obesity therapy is needed. The majority of current anti-obesity drugs are analogs of anorexigenic neurotransmitters, aiming to reduce food intake by either decreasing appetite or suppressing the craving for food. Unfortunately, their severe psychiatric or cardiovascular side effects have highlighted the need for alternative therapeutic strategies (for reviews, see Rodgers et al. 2012, Bray \& Ryan 2014, Manning et al. 2014). The ideal anti-obesity drug should produce sustained weight loss with minimal side effects. Recent progress in an understanding of peptidergic signaling of hunger and satiety, both from the gastrointestinal tract and its upstream pathways in the hypothalamus, have opened the possibility for using anorexigenic neuropeptides in obesity treatment (Arch 2015, Patel 2015).

The anorexigenic neuropeptide prolactin-releasing peptide (PrRP) was initially identified as a possible regulator of prolactin secretion from the anterior pituitary cells (Sun et al. 2005), and was finally isolated from the hypothalamus as a ligand for the human orphan G-protein-coupled receptor GPR10 (Hinuma et al. 1998). Recently, it has been established that PrRP has other physiological functions (Onaka et al. 2010), including the regulation of food intake (Lawrence et al. 2000) and energy expenditure (Takayanagi et al. 2008), whereas its involvement in the regulation of hypothalamic-pituitary-adrenal (HPA) axis (Dodd \& Luckman 2013) and its prolactin-releasing ability was questioned (Jarry et al. 2000). PrRP-producing cells are localized in the dorsomedial hypothalamic nucleus and in A1/A2 regions of the medulla oblongata (Yamada et al. 2009) in the brainstem. The fibers of these cells project to the paraventricular nucleus (PVN), the basal nucleus of the amygdala, and other regions throughout the brain (Yamada et al. 2009), suggesting that PrRP acts mainly in the central nervous system (CNS). It has been shown in rodents that intracerebroventricular injection of PrRP decreased food intake and body weight (Lawrence et al. 2002, Maixnerová et al. 2011). Moreover, mice deficient in Prrp or Prrp receptor are obese (Gu et al. 2004, Takayanagi et al. 2008).

GPR10 is widely expressed throughout the brain (especially in the reticular nucleus of the thalamus, hypothalamic paraventricular nucleus, periventricular nucleus, dorsomedial hypothalamic nucleus, NTS, and area postrema), in the anterior pituitary, and the adrenal medulla (reviewed in Onaka et al. 2010, Dodd \& Luckman 2013).
As PrRP is a centrally acting neuropeptide, it is difficult to administer it peripherally to induce its effect in the brain. Recently, we have modified PrRP by an attachment of longer fatty acids that allowed us to apply peptide to the periphery to achieve its central biological effect. As we have shown in our previous publication, the lipidization of PrRP resulted in the stabilization of the molecule, possibly by promoting the association of these peptides with circulating plasma proteins. We can also hypothesize that it enabled penetration of the molecule through the blood-brain barrier, as we observed a significant increase in c-Fos immunoreactivity in the hypothalamic and brainstem nuclei involved in food intake regulation after peripheral administration (Maletinska et al. 2015). However, there is still a lack of the direct proof of the lipidated PrRP entering the CNS as c-Fos could be reflective of either a direct or an indirect action of the compound in the CNS.

Various animal models are used to investigate the novel anti-obesity drugs (Vickers et al. 2011). The best animal obesity models are often diet-induced ones, as they result in changes consistent with those seen in obese patients (Vickers et al. 2011). Diet-induced obese (DIO) rats or mice are produced generally from lean animals that have free access to a diet high in fat over a period of 3-4 months. An increase in body weight occurs gradually, principally by a marked increase in body fat (Harrold \& Halford 2006, Madsen et al. 2010). Woods et al. (2003) showed that measuring body fat is a more sensitive criterion for assessing obesity in animals, as rats fed a high-fat diet (40\% of total calories) for 10 weeks displayed a $10 \%$ increase in total body weight but a 35-40\% increase in total body fat compared with the animals fed a standard diet. In addition to the DIO rat model, the Zucker diabetic rat model, which is a model with impaired leptin receptor signaling (Fellmann et al. 2013), is frequently used for studying the potential of anti-obesity and anti-diabetic peptidic drugs (Andreassen et al. 2014, Skarbaliene et al. 2015).

Recent studies have shown the efficiency of some peptidic drugs in either the DIO or ZDF rat model. Liraglutide, palmitoylated glucagon-like peptide (GLP1) analog, was proven to lower food intake and body weight after a chronic 12-week s.c. administration in DIO rats (Raun et al. 2007). Infusion of a combination of another GLP1 analog, exenatide, and a peptide YY 3-36 analog caused a reduction in food intake and body weight in DIO rats (Reidelberger et al. 2011). In the ZDF diabetic rat model, a combination of exenatide and gastrin treatment (Skarbaliene et al. 2015), as well as glucose-dependent insulinotropic peptide treatment (Tatarkiewicz et al. 2014),

Published by Bioscientifica Ltd 
caused a prolonged glucose-lowering effect rather than a body weight-decreasing effect. Finally, a study by Fosgerau et al. (2014) described that the biological effect of a novel selective lipidized analog of $\alpha$-melanocyte-stimulating hormone $(\alpha-\mathrm{MSH})$ with a strong central anorexigenic effect caused a significant decrease in food intake and body weight in DIO rats after repeated peripheral administration.

In this study, we used two rat models of obesity and diabetes (Sprague-Dawley rats fed a high-fat diet and the lack of function of leptin receptor in Zucker diabetic rats) to evaluate the chronic anti-obesity potency of our novel lipidized PrRP and the involvement of the leptin signaling pathway in its effects. We have recently shown in DIO mice that palm-PrRP31 decreased food intake and body weight and improved metabolic parameters associated with obesity and diabetes (Maletinska et al. 2015). Therefore, we compared food intake, body weight, and metabolic parameters in both rat models after chronic treatment with palm-PrRP31.

\section{Materials and methods}

\section{Peptides}

Palmitoylated PrRP analog palm-PrRP31 (N-palm-SRTHRH SMEIRTPDINPAWYASRGIRPVGRF- $\mathrm{NH}_{2}$ ) was synthesized and purified at the Institute of Organic Chemistry and Biochemistry, Prague, Czech Republic, as described previously (Blechová et al. 2013). Palmitoylation of PrRP was performed as described previously (Maletínská et al. 2012), on fully protected peptide on resin as the final step. The purity and identity of the peptide was determined by analytical HPLC and by using a Q-TOF micro MS technique (Waters, Milford, MA, USA).

\section{Chronic effect of palm-PrRP31 on body weight and biochemical parameters in DIO Sprague-Dawley rats and ZDF rats}

Animals and diets Sprague-Dawley male rats, 6-8 weeks old, were obtained from Harlan Laboratories (Correzzana, Italy). The animals were acclimatized for 1 week before initiation of the feeding the research diet. Rats were fed either the high-fat (HF) diet D12492 (60\% fat $\mathrm{kcal}, 20 \%$ carbohydrate $\mathrm{kcal}$, and $20 \%$ protein $\mathrm{kcal}$ ) or the low-fat (LF) diet D12450B (10\% fat kcal, 70\% carbohydrate $\mathrm{kcal}$, and 20\% protein kcal) (Research Diets Inc., New Brunswick, NJ, USA) and given water ad libitum for 25 weeks (weight-gaining period).
ZDF-Lepr ${ }^{\mathrm{ra}} / \mathrm{Crl}$, diabetic $f a / f a$ male rats, and lean controls, both 6 weeks old, were obtained from Charles River (Saint-Germain-sur-l'Arbresle, France) and acclimatized for 1 week before the start of the experiments. The rats were fed a diet of Purina 5008 (PMI Nutrition International, LLC, Richmond, IN, USA). During the dosing period, $50-55 \mathrm{~g}$ and $25-30 \mathrm{~g}$ of the diet were fed to diabetic and nondiabetic control rats, respectively.

The rats were housed under controlled conditions with a constant temperature of $22 \pm 2^{\circ} \mathrm{C}$, a relative humidity $45-65 \%$, and a fixed day/light cycle (06:00-18:00 h). All procedures and experimental protocols conformed to the European Convention on Animal Protection and Guidelines on Research Animal Use.

Study design and drug administration An overview of the studies design is provided in Fig. 1A (DIO Sprague-Dawley rats) and 1B (ZDF rats). After 24 weeks on the HF diet, 32 DIO Sprague-Dawley rats with the highest body weight (BW) were selected and divided into four experimental groups $(n=8)$ : (A) vehicle; (B) $0.2 \mathrm{mg} / \mathrm{kg}$ palm-PrRP31; (C) $1 \mathrm{mg} / \mathrm{kg}$ palm-PrRP31; and (D) $5 \mathrm{mg} / \mathrm{kg}$ palm-PrRP31. The doses used in this study were chosen according to previously tested food intake after acute intraperitoneal (IP) administration of palm-PrRP31 in rats (results not shown). Rats fed with the low-fat diet formed the control group $(n=8)$.

In ZDF rats, a baseline oral glucose tolerance test was performed in overnight fasted rats on days -5 and -4 . Randomization of the rats into the experimental groups was performed based on body weight and blood glucose levels (average body weight of $352.5 \pm 4.4 \mathrm{~g}$, average blood glucose level of $24.5 \pm 0.9 \mathrm{mmol} / \mathrm{L}$ ). The following experimental groups $(n=8)$ were established: (A) vehicle; (B) $1 \mathrm{mg} / \mathrm{kg}$ palm-PrRP31; and (C) $5 \mathrm{mg} / \mathrm{kg}$ palmPrRP31. Nondiabetic lean rats were used as controls $(n=8)$.

Palm-PrRP31 for IP administration was dissolved in $50 \mathrm{mM}$ phosphate buffered saline (PBS), $\mathrm{pH}=6$; LoBind vials and tips (Eppendorf AG, Hamburg, Germany) were used for the formulation. The peptide solutions were prepared fresh for single-day dosing and administered twice a day (07:00 h and 15:00 h) in a dosing volume of $1.0 \mathrm{~mL} / \mathrm{kg}$ IP for 17 days (dosing period). The HF diet-fed control group and the LF diet-fed control group were treated bi-daily with PBS in a dosing volume of $1.0 \mathrm{~mL} / \mathrm{kg}$ IP, as well as were the diabetic and nondiabetic controls.

The food intake (grams of food consumed) and body weight were measured daily during the dosing period, and the rats were fed the same diet as during the pre-dosing period. On days 16 and 17, an oral glucose tolerance

Published by Bioscientifica Ltd 
A

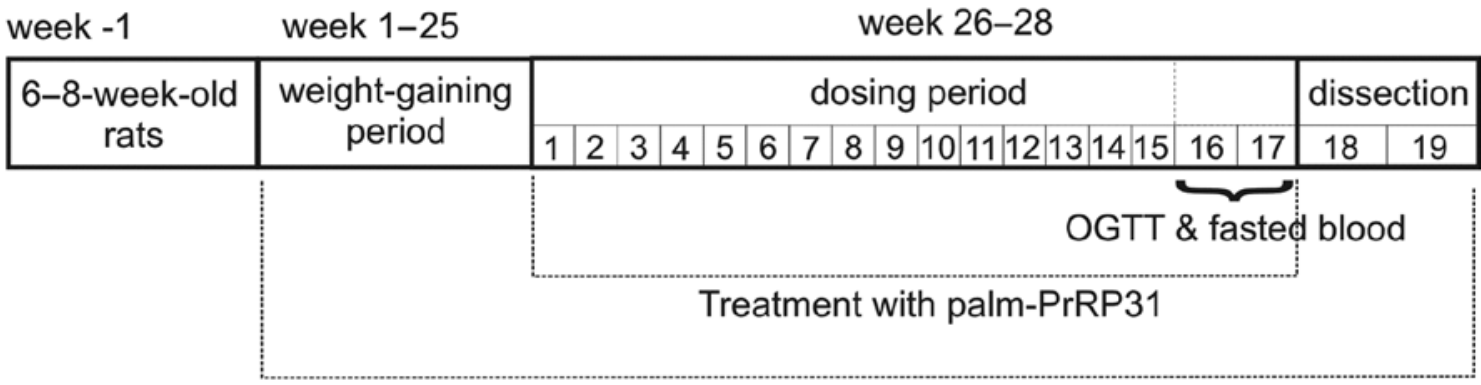

HF/LF feeding

B week -1 week 1-5 week 6-8

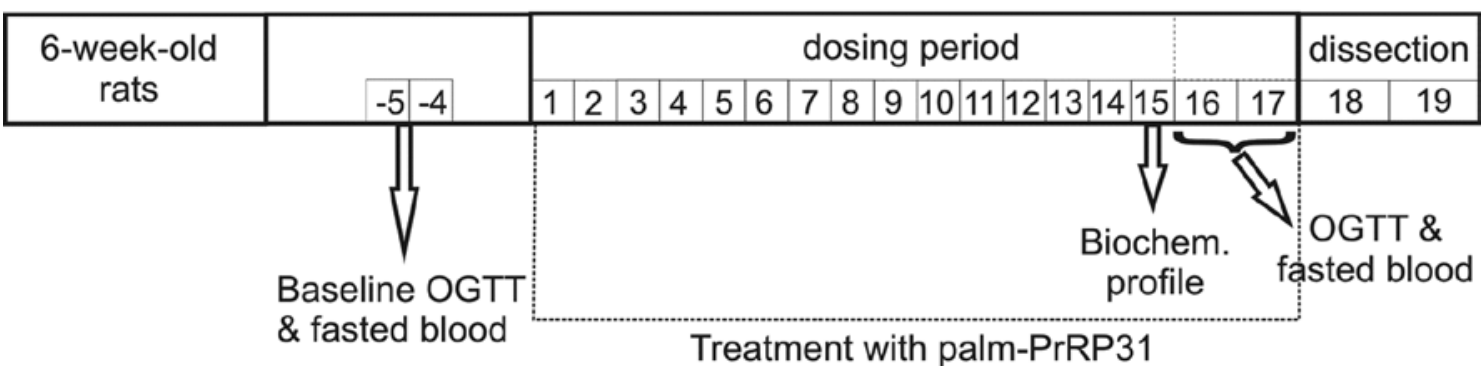

Figure 1

Study design. A schematic overview of the study design illustrating the treatment of DIO rats (A) and ZDF rats (B) with palm-PrRP31 and related sampling. OGTT, oral glucose tolerance test.

test was performed and therefore body weight and food intake were not measured. In ZDF rats, nonfasted blood samples were collected on day 15 for determination of the biochemical plasma profile.

Oral glucose tolerance test The oral glucose tolerance test (OGTT) was performed after overnight fasting on days 16 and 17. At time point $0(09: 00 \mathrm{~h})$, blood was drawn from the tail vein and the animals were loaded with 50\% glucose at a dose of $2 \mathrm{~g} / \mathrm{kg}$ perorally (PO) under slight isoflurane anesthesia. Blood samples were subsequently drawn from the tail vein into the heparinized capillaries at 15, 30, 60, 90, 120 and $180 \mathrm{~min}$ thereafter. The blood glucose concentrations were determined in whole blood by using the glucose oxidase method (glucose analyzer 8/28 BIOSEN S Line, EKF Diagnostics, Barleben, Germany).

Plasma profile: blood sampling and processing and biochemical analyses the blood samples for biochemical analyses were collected on days 16 and 17 from fasted isoflurane-anesthetized DIO
Sprague-Dawely rats before OGTT. The blood was collected from the orbital venous plexus into pre-cooled EDTA/NaF vacutainer tubes or EDTA Eppendorf tubes and centrifuged $\left(10,000 \mathrm{~g}, 5 \mathrm{~min}, 4^{\circ} \mathrm{C}\right)$ to prepare the plasma for measurement of free fatty acid (FFA), cholesterol, triglycerides, and leptin, respectively.

Before OGTT, $200 \mu \mathrm{L}$ of fasted blood was collected from the tail tip of ZDF rats into pre-cooled EDTA Multivette tubes and centrifuged $\left(10,000 \mathrm{~g}, 5 \mathrm{~min}, 4^{\circ} \mathrm{C}\right)$ to prepare the plasma for measurement of insulin level. The blood samples for other biochemical analyses were collected on day 15 from nonfasted ZDF rats. Blood samples were collected from the tail tip into pre-cooled EDTA/NaF vacutainer tubes or EDTA Multivette/EDTA Eppendorf tubes and centrifuged $\left(10,000 \mathrm{~g}, 5 \mathrm{~min}, 4^{\circ} \mathrm{C}\right)$ to prepare the plasma for FFA, cholesterol, triglycerides, and leptin measurements, respectively.

Blood plasma samples from both animal models were stored at $-20^{\circ} \mathrm{C}$ until analyses. Blood glucose was measured as described in the previous paragraph. Insulin level was determined using a commercial ultrasensitive rat insulin ELISA kit (Mercodia, Uppsala, Sweden). Cholesterol,

Published by Bioscientifica Ltd. 
triglycerides, and FFA were measured by the automatic analyzer Hitachi 912 (Boehringer Mannheim, Germany) using commercial kits (Roche Diagnostics $\mathrm{GmbH}$, Mannheim, Germany; FFA - Wako Chemicals GmbH, Germany). Leptin was determined using commercial mouse and rat leptin ELISA kits (Biovendor, Brno, Czech Republic).

Tissue dissection On days 18 and 19, the animals were killed by bleeding under isoflurane anesthesia. Four rats of each group were dissected within one day. Liver, epididymal (EF), perirenal (PF) and inguinal (IF) fat tissues were dissected from DIO Sprague-Dawley rats. Liver, EF and IF were dissected from ZDF rats. The tissue samples were weighed, frozen in liquid nitrogen, and stored at $-20^{\circ} \mathrm{C}$ until the tissue analyses were conducted.

Determination of mRNA expression The mRNA expressions were determined only in DIO Sprague-Dawley rats. Samples of adipose tissue (IF, EF) and liver were processed as described previously (Maletínská et al. 2011). Determination of the mRNA expression of genes of interest (acetyl-CoA carboxylase 1 (Acaca), fatty acid synthase (Fasn), lipoprotein lipase (Lpl), and fatty acid binding protein 4 (Fabp4) in IF and EF, and Acaca, Fasn and sterol regulatory element-binding transcription factor 1 (Srebf1) in liver was performed using an ABI PRISM 7500 instrument (Applied Biosystems). The expression of beta-glucuronidase (Gusb) was used to compensate for variations in input RNA amounts and the efficiency of reverse transcription.

\section{Statistical analyses}

The data are presented as the mean \pm s.e.m. Statistical analyses were performed by unpaired $t$-test, one-way ANOVA with Dunnett's post hoc test or repeated measures ANOVA with Bonferroni post hoc test at a 5\% level of probability in comparison with the control group, as indicated in the figure and table legends. The differences were considered significant at $P<0.05$. Where standard error bars are not visible in the figures, standard error was within the symbol size.

\section{Results}

\section{Characterization of the model of HF diet-induced obesity: HF vs LF diet-fed controls treated with vehicle}

The consumption of a HF diet resulted in a significantly affected body weight gain; at the start of the dosing period, the average BW of the LF diet-fed control group was $581.4 \pm 4.4 \mathrm{~g}$, whereas the average BW of the HF dietfed control group was $638.6 \pm 10.4 \mathrm{~g}(P<0.001)$. However, over the dosing period, the LF diet-fed controls consumed a significantly higher overall amount and caloric content of diet than those on the HF diet (Fig. 2A). In spite of this fact, the body weights of HF diet-fed controls were significantly higher than those of the LF diet-fed controls throughout the entire dosing period (Fig. 2B). At the end of the experiment, the LF and HF diet-fed controls showed a similar OGTT curve (Fig. 2C), whereas fasting glucose levels were increased in the HF diet-fed controls (Table 1A). The plasma insulin and FFA concentrations were slightly lower in HF diet-fed controls in comparison with the LF diet-fed controls, while the cholesterol and triglycerides plasma levels did not differ between these two control groups (Table 1A). The HF diet feeding did not result in enlargement of the liver but did lead to a markedly larger mass of epididymal and perirenal fat compared with the LF diet feeding, even though leptin levels did not significantly differ between the HF and LF diet-fed controls (Table 1A).

\section{Chronic effect of palm-PrRP31 on body weight and biochemical and metabolic parameters in DIO rats}

Food intake and body weight At the beginning of the dosing period, the mean BW and variance were similar among the groups of DIO rats (Table 1). The 17-day IP treatment with palm-PrRP31 lowered food intake in a dose-dependent manner, with the effect being more pronounced at week 1 and significant at 1 and $5 \mathrm{mg} / \mathrm{kg}$ doses (Fig. 3A). Similarly, body weight was reduced significantly in a dose-dependent way, and the most pronounced BW loss occurred after a 1-week treatment. The highest tested dose of palm-PrRP31 lowered BW by $8 \%$ (Fig. 3B).

\section{Oralglucosetolerancetest, biochemical parameters} and fat and liver weights Treatment with palmPrRP31 gave a small but statistically significant increase in fasting plasma glucose levels at the two highest doses in DIO rats (Table 1B). However, the treatment lowered final OGTT blood glucose levels in a dose-independent manner. This reduction was most pronounced after treatment with the $1 \mathrm{mg} / \mathrm{kg}$ dose of palm-PrRP31, which was significant compared with the vehicle-treated obese control group (Fig. 3C).

The palm-PrRP31 treatment did not affect fasted insulin plasma concentrations or cholesterol and 
A
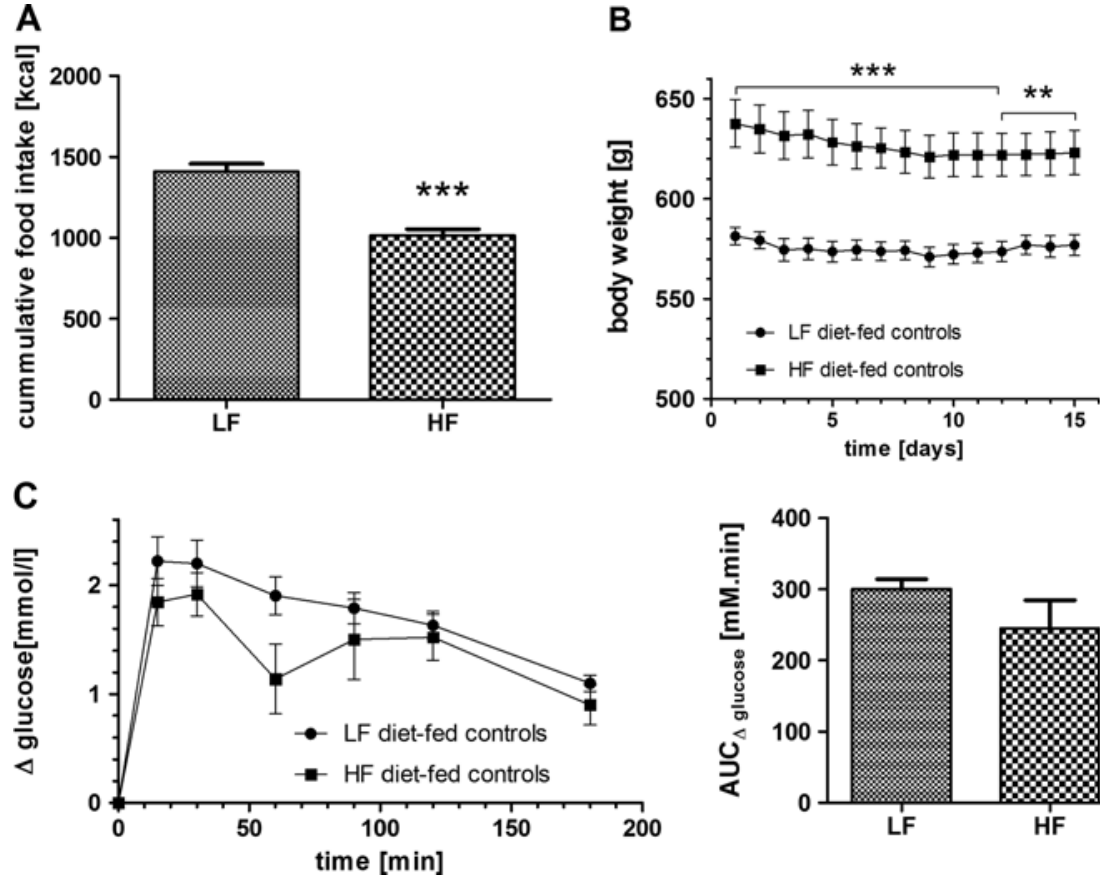

\section{Figure 2}

Characterization of a DIO model. The rats were fed either low-fat (LF) or high-fat (HF) diet for 25 weeks, starting from 6 to 8 weeks of age. For the following 17-day the dosing period, PBS was IP administered to rats ( $50 \mathrm{mM}$ PBS pH 6, $1 \mathrm{~mL} / \mathrm{kg}$ ). Food intake of both groups was monitored daily during the dosing period and is presented as a cummulative value (A), body weight was also monitored daily for 15 days (B). At the end of the experiment (on days 16-17), oral glucose tolerance test was performed; the results are shown as $\Delta$ glucose profile and $\mathrm{AUC}_{\Delta \mathrm{glucose}}$ (C). The data are presented as mean \pm S.E.M. Statistical analysis was performed by unpaired $t$-test ( $A$ and $C)$ or repeated measures ANOVA with Bonferroni post hoc test (B and $C$ ), significance is $* * P<0.01$, $* * * P<0.001$ vs the LF diet-fed control group $(n=7-8)$. triglycerides plasma levels. Palm-PrRP31 treatment nonsignificantly increased FFA plasma levels and nonsignificantly decreased plasma leptin levels at the highest dose (Table 1B). However, treatment with palmPrRP31 at the highest tested dose resulted only in a nonsignificant reduction in visceral and subcutaneous fat deposits and a mild reduction in liver weight (Table 2B).

mRNA expression in fat and liver of DIO rats In inguinal and epididymal adipose tissues, no significant changes induced by palm-PrRP31 treatment were found in the mRNA expression of genes involved in lipid metabolism, such as Acaca, Fasn, Lpl, and Fabp4 (results not shown).
However, the mRNA expression of Srebf1, Acaca, and Fasn were significantly decreased in the livers of DIO rats after treatment with the $5 \mathrm{mg} / \mathrm{kg}$ dose of palm-PrRP31 (Fig. 4).

\section{Characterization of diabetic ZDF rats: diabetic vs nondiabetic rats treated with vehicle}

The diabetic control rats consumed significantly higher amounts of food compared with the nondiabetic controls during the entire dosing period (Fig. 5A). During the dosing period, the difference in body weight between diabetic and nondiabetic rats diminished slightly, most likely due to the progressing diabetes in diabetic ZDF rats. The body weight gain of nondiabetic control rats was significantly

Table 1 Metabolic parameters analyzed in blood of DIO rats at the end of the experiment. (A) Comparison of LF and HF diet-fed control group, (B) Effect of the 17-day treatment of DIO rats with palm-PrRP31.

\begin{tabular}{|c|c|c|c|c|c|c|c|}
\hline & $\begin{array}{l}\text { Pre-treatment } \\
\text { body weight }(\mathrm{g})\end{array}$ & $\begin{array}{l}\text { Fast. glucose } \\
\quad(\mathrm{mmol} / \mathrm{L})\end{array}$ & Insulin (pmol/L) & $\begin{array}{l}\text { Leptin } \\
\text { (ng/mL) }\end{array}$ & FFA $(\mu \mathrm{mol} / \mathrm{L})$ & $\begin{array}{l}\text { Triglycerides } \\
\text { (mg/mL) }\end{array}$ & $\begin{array}{l}\text { Cholesterol } \\
(\mathrm{mmol} / \mathrm{L})\end{array}$ \\
\hline \multicolumn{8}{|l|}{ (A) Diet } \\
\hline LF & $581.4 \pm 4.4$ & $4.82 \pm 0.10$ & $138.92 \pm 14.05$ & $6.05 \pm 0.60$ & $479.13 \pm 55.5$ & $1.79 \pm 0.07$ & $2.64 \pm 0.12$ \\
\hline $\begin{array}{l}\text { HF } \\
\text { (B) Treatment }\end{array}$ & $638.6 \pm 10.4 * * *$ & $5.75 \pm 0.21$ ** & $103.51 \pm 16.48$ & $6.75 \pm 1.02$ & $367.43 \pm 46.12$ & $1.58 \pm 0.19$ & $2.77 \pm 0.11$ \\
\hline $\begin{array}{l}\text { Vehicle } \\
\text { palm-PrRP31 } 0.2 \mathrm{mg} / \mathrm{kg}\end{array}$ & $\begin{array}{l}638.6 \pm 10.4 \\
646.4 \pm 10.6\end{array}$ & $\begin{array}{l}5.75 \pm 0.21 \\
5.72 \pm 0.19\end{array}$ & $\begin{array}{c}103.51 \pm 16.48 \\
79.88 \pm 8.80\end{array}$ & $\begin{array}{l}6.75 \pm 1.02 \\
6.08 \pm 0.92\end{array}$ & $\begin{array}{l}367.43 \pm 46.12 \\
383.75 \pm 30.76\end{array}$ & $\begin{array}{l}1.58 \pm 0.19 \\
1.72 \pm 0.12\end{array}$ & $\begin{array}{l}2.77 \pm 0.11 \\
2.63 \pm 0.14\end{array}$ \\
\hline palm-PrRP31 $1 \mathrm{mg} / \mathrm{kg}$ & $647.8 \pm 12.5$ & $6.99 \pm 0.21 * *$ & $95.40 \pm 12.83$ & $5.04 \pm 1.02$ & $371.88 \pm 38.28$ & $1.74 \pm 0.09$ & $2.73 \pm 0.07$ \\
\hline palm-PrRP31 $5 \mathrm{mg} / \mathrm{kg}$ & $637.8 \pm 12.7$ & $7.43 \pm 0.42 * * *$ & $119.31 \pm 18.12$ & $4.71 \pm 0.64$ & $401.25 \pm 35.14$ & $1.58 \pm 0.07$ & $2.48 \pm 0.10$ \\
\hline
\end{tabular}

Data are presented as mean \pm S.E.M. Statistical analysis was performed by unpaired $t$-test (A) or one-way ANOVA with Dunnett's post hoc test (B). Significance is $* * P<0.01, * * * P<0.001$ vs the LF diet-fed control group (A).

FFA, free fatty acids; HF, high-fat diet; LF, low-fat diet. 

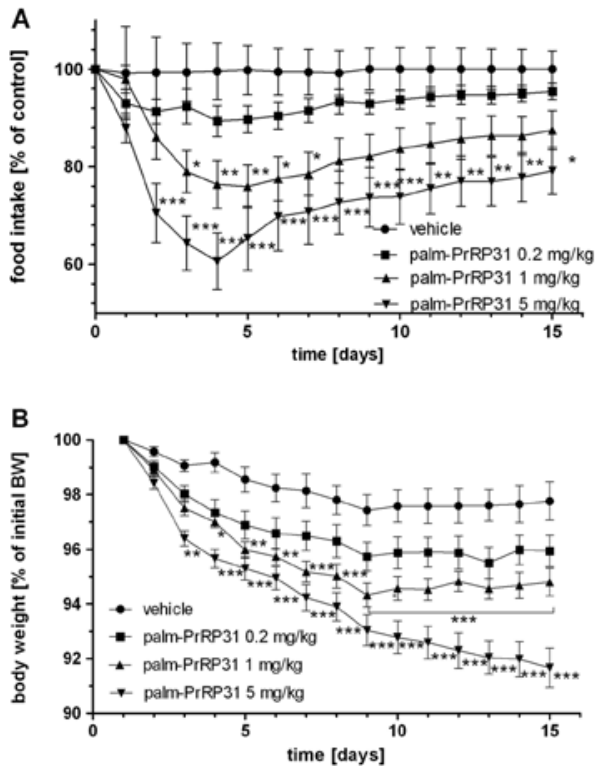
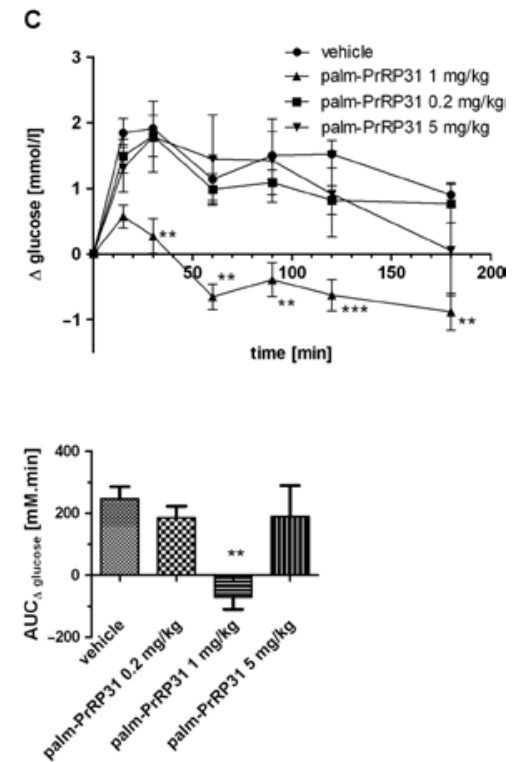

Figure 3

Chronic effect of palm-PrRP31 on food intake (A), body weight (B), and OGTT response (C) in DIO rats. Palm-PrPR31 was administered IP at doses of $0.2,1$, and $5 \mathrm{mg} / \mathrm{kg}$ (dissolved in $50 \mathrm{mM} \mathrm{PBS}, \mathrm{pH} 6$ ) twice a day for 17 days. Food intake and body weight were monitored daily for 15 days; food intake is expressed as a percentage of food intake in the vehicle-treated control group, body weight is expressed as a percentage of the initial body weight. OGTT was performed on days 16 and 17 and its results are shown as $\Delta$ glucose profile and $\mathrm{AUC}_{\Delta \text { glucose. }}$. Data are presented as mean \pm S.E.M. Statistical analysis was performed by repeated measures ANOVA with Bonferroni post hoc test $(\mathrm{A}, \mathrm{B}$, and $\mathrm{C})$ or one-way ANOVA with Dunnett's post hoc test (C), significance is $* P<0.05, * * P<0.01, * * * P<0.001$ vs the vehicle-treated obese control group $(n=7-8)$. higher than that of diabetic ZDF controls, despite the fact that diabetic ZDF controls were hyperphagic (Fig. 5B).

During the dosing period, the diabetic control rats injected with vehicle showed a high relatively invariable hyperglycemia and exhibited markedly lowered glucose tolerance during OGTT in comparison with nondiabetic control animals at the end of the dosing period (Fig. 5C). The plasma insulin levels of control diabetic rats were nonsignificantly higher than those of the nondiabetic controls at the end of the dosing period, the fasting glucose levels were significantly increased in control diabetic rats (Table $3 \mathrm{~A}$ ).

The diabetic rats showed severe hyperlipidemia at the end of the dosing periods. The plasma cholesterol and triglycerides concentrations in diabetic rats were significantly increased in comparison with nondiabetic controls. The plasma concentrations of free fatty acids were nonsignificantly elevated in diabetic rats (Table $3 \mathrm{~A}$ ).

At the end of the dosing period, the plasma leptin levels of control diabetic rats were significantly higher than those of the nondiabetic controls. The diabetic rats developed obesity and hepatomegaly. The liver enlargement and depots of inguinal and epididymal fats were significantly higher in comparison with nondiabetic control animals (Table 4A).

\section{Chronic effect of palm-PrRP31 on body weight and biochemical and metabolic parameters in ZDF rats}

Food intake and body weight The IP treatment of diabetic ZDF rats with palm-PrPR31 dose-dependently lowered food intake, with a significant effect at the $5 \mathrm{mg} / \mathrm{kg}$

Table 2 Liver and adipose tissue weights in DIO rats at the end of the experiment. (A) Comparison of LF and HF diet-fed control group. (B) Effect of the 17-day treatment of DIO rats with palm-PrRP31.

\begin{tabular}{|c|c|c|c|c|}
\hline & Epidid. fat (\% of BW) & Perirenal fat ( $\%$ of BW) & Inguinal fat ( $\%$ of $B W$ ) & Liver ( $\%$ of BW) \\
\hline \multicolumn{5}{|l|}{ (A) Diet } \\
\hline LF & $0.93 \pm 0.04$ & $0.89 \pm 0.05$ & $1.3 \pm 0.06$ & $16.33 \pm 0.30$ \\
\hline $\mathrm{HF}$ & $1.17 \pm 0.04 * * *$ & $1.49 \pm 0.12 * * *$ & $1.55 \pm 0.18$ & $15.31 \pm 0.43$ \\
\hline \multicolumn{5}{|l|}{ (B) Treatment } \\
\hline Vehicle & $1.17 \pm 0.04$ & $1.49 \pm 0.12$ & $1.55 \pm 0.18$ & $15.31 \pm 0.43$ \\
\hline palm-PrRP31 0.2 mg/kg & $1.19 \pm 0.07$ & $1.40 \pm 0.10$ & $1.65 \pm 0.12$ & $15.14 \pm 0.43$ \\
\hline palm-PrRP31 $1 \mathrm{mg} / \mathrm{kg}$ & $1.32 \pm 0.05$ & $1.40 \pm 0.07$ & $1.70 \pm 0.06$ & $15.64 \pm 0.46$ \\
\hline palm-PrRP31 5 mg/kg & $1.07 \pm 0.09$ & $1.24 \pm 0.10$ & $1.52 \pm 0.11$ & $14.81 \pm 0.37$ \\
\hline
\end{tabular}

Data are presented as mean \pm S.E.M. Statistical analysis was performed by unpaired $t$-test (A) or one-way ANOVA with Dunnett's post hoc test (B). Significance is $* * * P<0.001$ vs the LF diet-fed control group (A) or the HF diet-fed control group treated with vehicle (B). BW, body weight; HF, high-fat diet; LF, low-fat diet. 
A

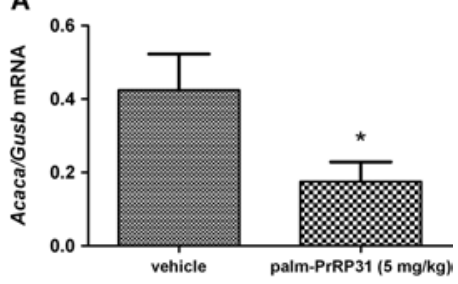

B
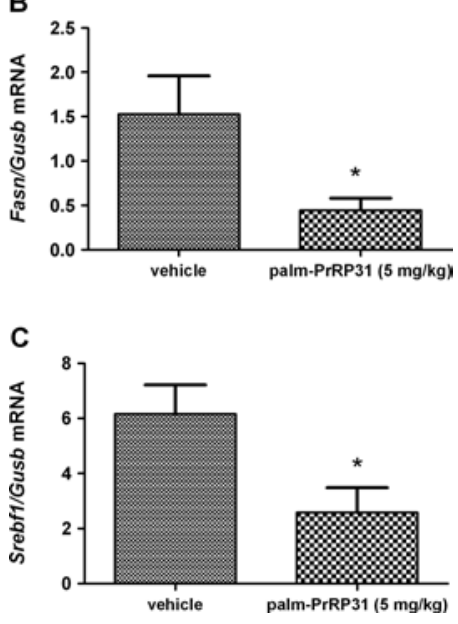

Figure 4

Chronic effect of palm-PrRP31 on mRNA expressions of Acaca (A), Fasn (B) and Srebf1 (C) in the liver of DIO rats. mRNA expressions were determined after the 17-day treatment with palm-PrPR31 at a dose of $5 \mathrm{mg} / \mathrm{kg}$. Data are presented as mean \pm s.E.M. The data were normalized to GUSB and analyzed by unpaired $t$-test, significance is $* P<0.05$ vs the vehicle-treated obese control group $(n=7-8)$.

dose (Fig. 6A). All rats were gaining weight during the dosing period. However, the body weight gain was not significantly lowered after the palm-PrRP31 treatment (Fig. 6B).

\section{Oral glucose tolerance test, biochemical parameters, and fat and liver weights The treat-} ment with palm-PrRP31 resulted in a nonsignificant dose-dependent decrease in blood glycemia during OGTT (Fig. 6C). Fasting glucose levels did not change significantly after the palm-PrRP31 treatment. Palm-PrRP31 treatment significantly and dose-dependently decreased plasma cholesterol and nonsignificantly decreased plasma FFA, triglycerides, leptin and insulin levels (Table 3B). The weights of liver or fat masses were not significantly changed by the treatment (Table 4B).

\section{Discussion}

In terms of pharmacotherapy for obesity, only a few new drugs have been registered over the last few years
(Arch 2015, Patel 2015). Despite the many known peptidic hormones involved in food intake regulation, only one of them, liraglutide, a peptidic drug acting through the GLP1 receptor, has recently been approved for anti-obesity treatment. Therefore, novel drugs acting through other pathways are needed. Thus, the anorexigenic PrRP with its GPR10 receptor represents a promising new candidate.

We have recently shown that novel lipidized PrRP analogs are potential anti-obesity agents that are able to exert their central effect after peripheral administration (Maletinska et al. 2015). The anorexigenic effect of palmPrRP31, both acute in lean mice and chronic in DIO mice, was demonstrated in our previous study (Maletinska et al. 2015). As the effects of various drugs may differ in different species, in this study we investigated the effect of palm-PrRP31 on lowering food intake and body weight in two rat models: Sprague-Dawley rats with DIO, a model of obesity and insulin resistance, and diabetic ZDF rats, selected from fatty Zucker rats with severe insulin resistance and a lack of leptin signaling (Vickers et al. 2011). We aimed to investigate if palm-PrRP31 action depends on the presence of leptin receptor signaling.

The common feature observed in DIO rats and ZDF rats is hyperleptinemia. However, the origin of excessive levels of circulating leptin is different. In rats with DIO, the growing adipose tissue mass secretes increasing amounts of leptin that gradually leads to dysregulation of its feedback on energy homeostasis and results in fat accumulation and a leptin-resistant state. In ZDF rats, similar to their progenitor fatty Zucker rats, a high level of circulating leptin results from the absence of a functional leptin receptor (Iida et al. 1996, Phillips et al. 1996). In ZDF rats, leptin does not regulate food intake because its signaling is completely disabled due to a nonfunctional receptor.

In our study, as expected, the leptin levels of diabetic ZDF controls displayed several fold differences compared with those of the nondiabetic controls, as did the weights of their inguinal and epididymal fat and liver. In contrast, leptin levels in the HF diet-fed controls were not enhanced significantly compared with the LF diet-fed controls, even though the weights of their epididymal and perirenal fat were significantly higher.

The total lack of leptin signaling in ZDF rats is the reason for a severe insulin resistance; it is proven by the presence of hyperinsulinemia at such a young age (Etgen $\&$ Oldham 2000). Insulin resistance is further enhanced by feeding with a diet containing $6.5 \%$ fat, which is recommended for ZDF rats. Leptin ineffectiveness and slightly increased fat intake are the major and minor obesity causes, respectively (Etgen \& Oldham 2000,

Published by Bioscientifica Ltd. 
A
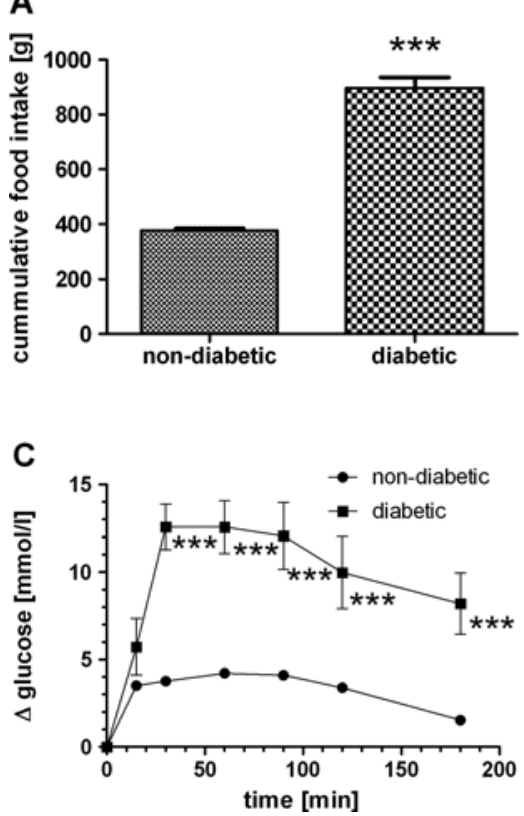

B
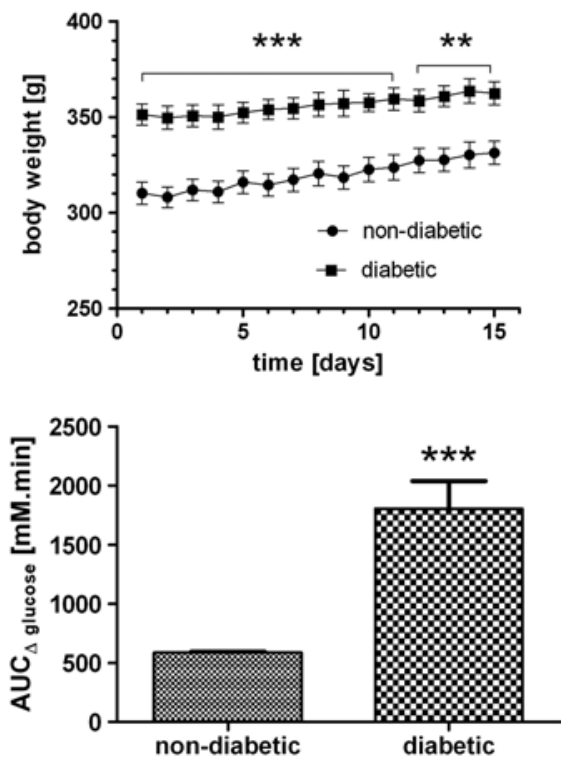

Figure 5

Characterization of diabetic ZDF rats. Diabetic fal fa ZDF rats and nondiabetic ZDF control rats were IP treated with PBS for 17 days (50 mM PBS pH 6, $1 \mathrm{~mL} / \mathrm{kg}$ ). Food intake of both groups was monitored daily during the dosing period and is presented as a cummulative value (A), body weight was also monitored daily for 15 days (B). At the end of the experiment (on days 16-17), oral glucose tolerance test was performed; the results are shown as $\Delta$ glucose profile and $\mathrm{AUC}_{\Delta}$ glucose (C). Data are presented as mean \pm S.E.M. Statistical analysis was performed by unpaired $t$-test ( $A$ and $C$ ) or repeated measures ANOVA with Bonferroni post hoc test (B and $C$ ), significance is $* * P<0.01, * * * P<0.001$ vs the nondiabetic control group $(n=8)$.
Sturis et al. 2003). Under obese conditions, central insulin resistance develops, as proopiomelanocortin (POMC) neurons in the hypothalamus do not respond to insulin to attenuate food intake and body weight (Konner \& Bruning 2012).

At the end of the palm-PrRP31 dosing period, diabetic ZDF controls had a higher cumulative food intake but a lower increase in body weight than the nondiabetic controls, pointing to a typical diabetic condition. In contrast, the HF diet-fed controls consumed less calories than the controls on the LF diet and their body weight dropped gradually but was significantly higher through the entire dosing period than that of LF fed controls, which did not change.

A 2-week intraperitoneal treatment with palm-PrRP31 resulted in a significantly reduced food intake and body weight in DIO rats, with this effect increasing as the dose administered increased. The palm-PrRP31 already caused a significant effect at the $1 \mathrm{mg} / \mathrm{kg}$ dose. In our study, food intake in the HF fed group administered $5 \mathrm{mg} / \mathrm{kg}$ of palmPrRP31 twice daily decreased on several days by $40 \%$ and $24 \%$ after a 2-week-long treatment, compared with the HF diet-fed control group treated with vehicle. The body weight decrease corresponded to a decrease in food intake in the DIO rat model. A body weight change of $8 \%$ at the end of the palm-PrRP31 treatment in our DIO model was similar to that previously described after chronic treatment in the DIO rat model with the GLP1 analogs liraglutide (Raun et al. 2007, Madsen et al. 2010, Hayes et al. 2011) and exenatide (Reidelberger et al. 2011) or with an $\alpha$-MSH analog (Fosgerau et al. 2014). During the palm-PrRP31 treatment we did not observe any signs of

Table 3 Metabolic parameters analyzed in the blood of ZDF rats at the end of the experiment. (A) Comparison of nondiabetic and diabetic control group. (B) Effect of the 17-day treatment of diabetic ZDF rats with palm-PrRP31.

\begin{tabular}{|c|c|c|c|c|c|c|}
\hline & $\begin{array}{l}\text { Fasting glucose } \\
(\mathrm{mmol} / \mathrm{L})\end{array}$ & Insulin (pmol/L) & Leptin (ng/mL) & FFA $(\mu \mathrm{mol} / \mathrm{L})$ & $\begin{array}{l}\text { Triglycerides } \\
(\mathrm{mg} / \mathrm{mL})\end{array}$ & $\begin{array}{c}\text { Cholesterol } \\
(\mathrm{mmol} / \mathrm{L})\end{array}$ \\
\hline \multicolumn{7}{|c|}{ (A) Nondiabetic $\times$ diabetic } \\
\hline Nondiabetic & $4.43 \pm 1.10$ & $124.08 \pm 12.92$ & $5.58 \pm 0.67$ & $445.6 \pm 33.5$ & $1.28 \pm 0.16$ & $2.13 \pm 0.04$ \\
\hline $\begin{array}{l}\text { Diabetic } \\
\text { (B) Treatment }\end{array}$ & $16.42 \pm 1.82 * * *$ & $163.71 \pm 27.77$ & $16.58 \pm 1.38 * * *$ & $507.4 \pm 64.4$ & $6.26 \pm 1.75^{*}$ & $3.79 \pm 0.30 * * *$ \\
\hline Vehicle & $16.42 \pm 1.82$ & $163.71 \pm 27.77$ & $16.58 \pm 1.38$ & $507.4 \pm 64.4$ & $6.26 \pm 1.75$ & $3.79 \pm 0.30$ \\
\hline palm-PrRP31 $1 \mathrm{mg} / \mathrm{kg}$ & $18.80 \pm 1.08$ & $117.55 \pm 8.38$ & $13.29 \pm 0.92$ & $347.9 \pm 43.1$ & $4.72 \pm 0.47$ & $3.10 \pm 0.12 *$ \\
\hline palm-PrRP31 $5 \mathrm{mg} / \mathrm{kg}$ & $18.99 \pm 1.80$ & $105.75 \pm 19.89$ & $16.56 \pm 4.84$ & $478.5 \pm 49.6$ & $5.53 \pm 0.53$ & $2.91 \pm 0.12 * *$ \\
\hline
\end{tabular}

Data are presented as mean \pm S.E.M. Statistical analysis was performed by unpaired $t$-test (A) or one-way ANOVA with Dunnett's post hoc test (B). Significance is $* P<0.05, * * P<0.01, * * * P<0.001$ vs the nondiabetic control group (A) or the diabetic control group treated with vehicle (B). FFA, free fatty acids.

http://joe.endocrinology-journals.org DOI: 10.1530/JOE-15-0519 (c) 2016 Society for Endocrinology Printed in Great Britain
Published by Bioscientifica Ltd. 
Table 4 Liver and adipose tissue weights in ZDF rats at the end of the experiment. (A) Comparison of nondiabetic and diabetic control group. (B) Effect of the 17-day treatment of diabetic ZDF rats with palm-PrRP31.

\begin{tabular}{l} 
\\
\hline (A) Nondiabetic $\times$ diabetic \\
Nondiabetic \\
Diabetic \\
(B) Treatment \\
Vehicle \\
palm-PrRP31 $1 \mathrm{mg} / \mathrm{kg}$ \\
palm-PrRP31 $5 \mathrm{mg} / \mathrm{kg}$
\end{tabular}

\begin{tabular}{c}
\hline Epidid. fat $(\%$ of $B W)$ \\
$0.47 \pm 0.02$ \\
$0.91 \pm 0.04 * * *$ \\
\\
$0.91 \pm 0.04$ \\
$0.92 \pm 0.03$ \\
$0.94 \pm 0.02$
\end{tabular}

\begin{tabular}{c}
\hline Inguinal fat $(\%$ of $B W)$ \\
\\
$1.00 \pm 0.07$ \\
$2.85 \pm 0.14 * * *$ \\
\\
$2.85 \pm 0.14$ \\
$2.70 \pm 0.16$ \\
$2.69 \pm 0.23$
\end{tabular}

\begin{tabular}{|c|}
\hline Liver ( $\%$ of BW) \\
\hline $\begin{array}{c}9.78 \pm 0.28 \\
16.59 \pm 0.56^{* * *}\end{array}$ \\
\hline $\begin{array}{l}16.59 \pm 0.56 \\
15.84 \pm 0.57 \\
17.03 \pm 0.60\end{array}$ \\
\hline
\end{tabular}

Data are presented as mean \pm S.E.M. Statistical analysis was performed by unpaired $t$-test (A) or one-way ANOVA with Dunnett's post hoc test (B). Significance is $* * * P<0.001$ vs the nondiabetic control group (A) or the diabetic control group treated with vehicle (B). BW, body weight.

discomfort, nausea, abnormal behavior nor pathological changes at any dose used. Thus, palm-PrRP31 reveals a very promising body weight-lowering activity. Future work toward a better formulation of the drug could help to decrease the effective dose and increase the release of the compound both after intraperitoneal and subcutaneous administrations.

The palm-PrRP31 treatment in HF diet-fed rats caused only a tendency toward decreasing fat weight and leptin levels, which was not significant. Similarly, FFA levels were not significantly changed in palmPrRP31-treated rats on the HF diet. Despite this, the liver mRNAs of the enzymes catalyzing the de novo synthesis of fatty acids, Acaca and Fasn, were reduced significantly and in parallel with a reduction in the mRNA of Srebp1, their common transcription factor (Xiao \& Song 2013). The precise mechanism of the palm-PrRP31 attenuating effect on lipogenesis in the liver is not known, but a similar effect was shown in our previous study in DIO mice, where a 2-week-long palm-PrRP31 treatment attenuated the liver mRNAs of Acaca, Fasn, and Srebp1 (Maletinska et al. 2015) as well.

Even though food intake in diabetic ZDF rats in this study decreased from the first day of the palm-PrRP31 treatment, their body weight did not change. The most probable reason was that ZDF rats at age of 11 weeks were still growing, but starting the treatment at this age was necessary for a proper modeling of type 2 diabetes. Food intake was significantly decreased by treatment with a $5 \mathrm{mg} / \mathrm{kg}$ dose of palm-PrRP31 and dropped by $15-20 \%$ compared with the diabetic control vehicle-treated group. In our previous unpublished study, we did not find any effect of a 2-week-long SC treatment of palmPrRP31 on the body weight of 10 -week-old diabetic $d b / d b$
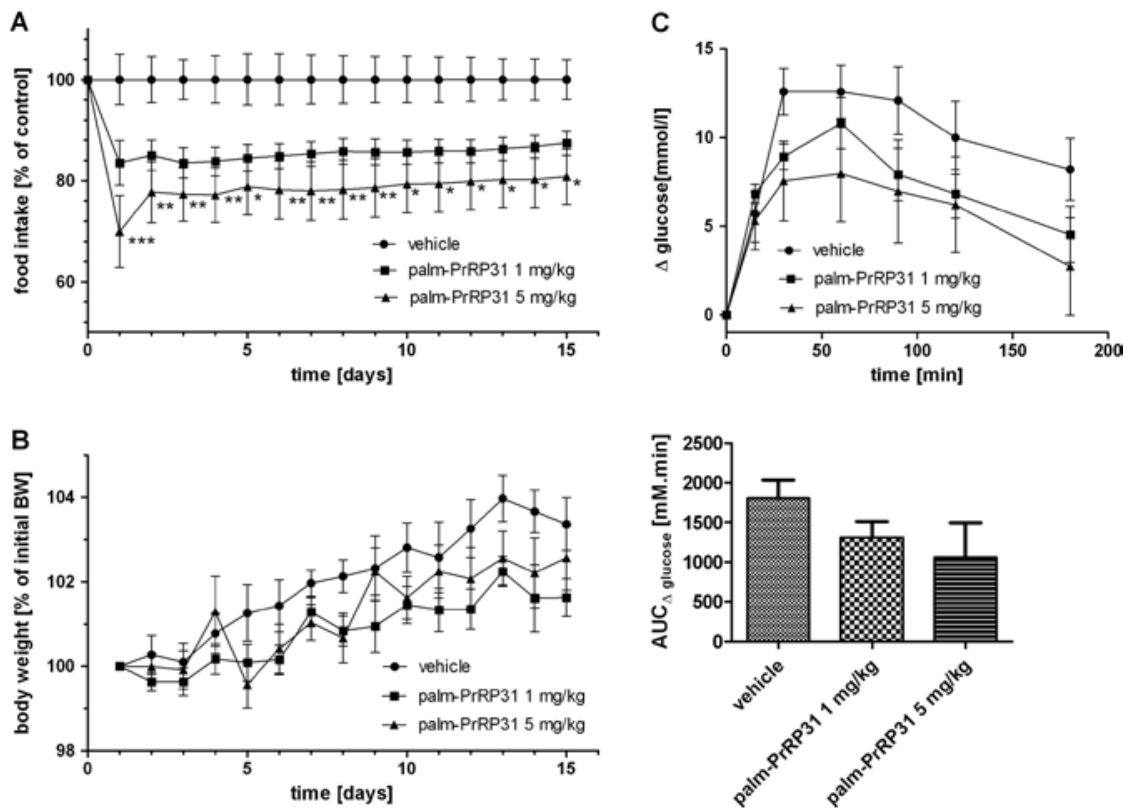

\section{Figure 6}

Chronic effect of palm-PrRP31 on food intake, body weight, and OGTT response in diabetic ZDF rats. Palm-PrPR31 was administered IP at doses of 1 and $5 \mathrm{mg} / \mathrm{kg}$ (dissolved in $50 \mathrm{mM}$ PBS, $\mathrm{pH} 6$ ) twice a day for 17 days. Food intake and body weight were monitored daily for 15 days; food intake is expressed as a percentage of food intake in the vehicle-treated control group, body weight is expressed as a percentage of the initial body weight. OGTT was performed on days 16 and 17 and its results are shown as $\Delta$ glucose profile and AUC $_{\Delta \text { glucose }}$. Data are presented as mean \pm S.E.M. Statistical analysis was performed by repeated measures ANOVA with Bonferroni post hoc test ( $A, B$, and $C$ ) or one-way ANOVA with Dunnett's post hoc test $(C)$, significance is $* P<0.05$, $\star * P<0.01, * * * P<0.001$ vs the vehicle-treated diabetic control group $(n=8)$. 
mice lacking a functional leptin receptor, although an attenuated final glucose level of OGTT was observed. In this study, in ZDF diabetic rats, palm-PrRP31 tended to lower the OGTT curves, but the results did not reach significance. No effects of palm-PrRP31 on fat and liver weight, insulin or leptin levels were found in the diabetic rats. The primary effect of palm-PrRP31 was anorexigenic and occurred both in DIO and ZDF diabetic rats. However, in ZDF rats, a deficiency in the functional leptin receptor could cause diminished PrRP efficacy, as the synergism of leptin and the PrRP anorexigenic effect is well known (Ellacott et al. 2002).

In our study, a significant glucose-lowering effect of palm-PrRP31 was found in DIO rats after the OGTT test. However, at the moment we are not able to explain the observed nonlinear relarionship in a satisfactory manner, but a possible palm-PrRP31 anti-diabetic effect should be studied in the future, as well as the surprising small but significant increase in fasting glucose levels. GLP1 analogs have been shown to exert their anti-diabetic effect in ZDF rats (Sturis et al. 2003, Vrang et al. 2012, Skarbaliene et al. 2015). As GLP1 is both an insulin-secreting promotor and a glucagon-secreting inhibitor (Willms et al. 1996), it should be determined if it is essential to induce insulin or attenuate glucagon secretion in ZDF rats to have an antidiabetic effect. Neither of these two properties has been attributed to palm-PrRP31 yet.

In conclusion, this study demonstrated that DIO rats that received a 2-week-long peripheral treatment with a palm-PrRP31 analog showed significantly decreased food intake and body weight, with a tendency toward leptin and fat depot reduction. This treatment was also associated with an improvement in glucose tolerance and the effect was caused at least partially by an attenuating effect on lipogenesis. In contrast, despite a food intakelowering effect, palm-PrRP31 failed to decrease body weight or improve glucose tolerance in ZDF rats, probably due to a lack of functional leptin receptor and therefore preventing an interaction of leptin and palm-PrRP31 in this rat model. Thus, GPR10 agonism is a promising target for the treatment of obesity, with palm-PrRP31 showing a high anorexigenic efficacy after peripheral administration.

\section{Declaration of interest}

The authors declare that there is no conflict of interest that could be perceived as prejudicing the impartiality of the research reported.

\section{Funding}

This work was supported by TA CR grant No. TE01020028, GA CR grant No. 15-08679S, RVO:61388963 of AS CR and RVO:67985823 of AS CR.

\section{Author contribution statement}

$V P$, J K, B Ž, and L M designed the studies. V P, J S, M H, J Z, and B M performed the studies and evaluated results. $M H, J K, B Z \check{Z}$, and $L M$ wrote the manuscript and all authors edited the manuscript.

\section{References}

Andreassen KV, Feigh M, HjulerST, Gydesen S, Henriksen JE, Beck-Nielsen H, Christiansen C, Karsdal MA \& Henriksen K 2014 A novel oral dual amylin and calcitonin receptor agonist (KBP-042) exerts antiobesity and antidiabetic effects in rats. American Journal of Physiology: Endocrinology and Metabolism 307 E24-E33. (doi:10.1152/ ajpendo.00121.2014)

Arch JR 2015 Horizons in the pharmacotherapy of obesity. Current Obesity Reports 4 451-459. (doi:10.1007/s13679-015-0177-4)

Blechová M, Nagelová V, Záková L, Demianová Z, Zelezná B \& Maletínská L 2013 New analogs of the CART peptide with anorexigenic potency: the importance of individual disulfide bridges. Peptides 39 138-144. (doi:10.1016/j.peptides.2012.09.033)

Bray GA \& Ryan DH 2014 Update on obesity pharmacotherapy. Annals of the New York Academy of Sciences 1311 1-13. (doi:10.1111/nyas.12328)

DoddGT \& Luckman SM 2013 Physiological roles of GPR10 and PrRP signaling. Frontiers in Endocrinology 4 20. (doi:10.3389/ fendo.2013.00020)

Ellacott K, Lawrence C, Rothwell N \& Luckman S 2002 PRL-releasing peptide interacts with leptin to reduce food intake and body weight. Endocrinology 143 368-374. (doi:10.1210/endo.143.2.8608)

Etgen GJ \& Oldham BA 2000 Profiling of Zucker diabetic fatty rats in their progression to the overt diabetic state. Metabolism 49 684-688. (doi:10.1016/S0026-0495(00)80049-9)

Fellmann L, Nascimento AR, Tibiriça E \& Bousquet P 2013 Murine models for pharmacological studies of the metabolic syndrome. Pharmacology \& Therapeutics 137 331-340. (doi:10.1016/j. pharmthera.2012.11.004)

Fosgerau K, Raun K, Nilsson C, DahlK \& Wulff BS 2014 Novel alpha-MSH analog causes weight loss in obese rats and minipigs and improves insulin sensitivity. Journal of Endocrinology 220 97-107. (doi:10.1530/ JOE-13-0284)

Gu W, Geddes B, Zhang C, Foley K \& Stricker-Krongrad A 2004 The prolactin-releasing peptide receptor (GPR10) regulates body weight homeostasis in mice. Journal of Molecular Neuroscience 22 93-103. (doi:10.1385/jmn:22:1-2:93)

HarroldJ \& HalfordJ 2006 The hypothalamus and obesity. Recent Patents on CNS Drug Discovery 1 305-314. (doi:10.2174/157488906778773616)

Hayes MR, KanoskiSE, Alhadeff AL \& Grill HJ 2011 Comparative effects of the long-acting GLP-1 receptor ligands, liraglutide and exendin-4, on food intake and body weight suppression in rats. Obesity 19 1342-1349. (doi:10.1038/oby.2011.50)

HinumaS, Habata Y, Fujii R, Kawamata Y, Hosoya M, FukusumiS, Kitada C, MasuoY, Asano T, Matsumoto H, et al. 1998 A prolactin-releasing peptide in the brain. Nature 393 272-276. (doi:10.1038/30515)

Iida M, Murakami T, Ishida K, Mizuno A, Kuwajima M \& Shima K 1996 Phenotype-linked amino acid alteration in leptin receptor cDNA from Zucker fatty (fa/fa) rat. Biochemical and Biophysical Research Communications 222 19-26. (doi:10.1006/bbrc.1996.0691)

Jarry H, Heuer H, Schomburg L \& Bauer K 2000 Prolactin-releasing peptides do not stimulate prolactin release in vivo. Neuroendocrinology 71 262-267. (doi:10.1159/000054544)

Konner AC \& BruningJC 2012 Selective insulin and leptin resistance in metabolic disorders. Cell Metabolism 16 144-152. (doi:10.1016/j. cmet.2012.07.004)

Lawrence C, Celsi F, Brennand J \& LuckmanS 2000 Alternative role for prolactin-releasing peptide in the regulation of food intake. Nature Neuroscience 3 645-646. (doi:10.1038/76597) http://joe.endocrinology-journals.org

DOI: $10.1530 /$ JOE-15-0519
(C) 2016 Society for Endocrinology Printed in Great Britain
Published by Bioscientifica Ltd. 
Lawrence C, Ellacott K \& Luckman S 2002 PRL-releasing peptide reduces food intake and may mediate satiety signaling. Endocrinology 143 360-367. (doi:10.1210/endo.143.2.8609)

MadsenAN, Hansen G, PaulsenSJ, LykkegaardK, Tang-Christensen M, HansenHS, Levin BE, LarsenPJ, KnudsenLB, FosgerauK, et al. 2010 Long-term characterization of the diet-induced obese and diet-resistant rat model: a polygenetic rat model mimicking the human obesity syndrome. Journal of Endocrinology 206 287-296. (doi:10.1677/JOE-10-0004)

Maixnerová J, Špolcová A, Pýchová M, Blechová M, Elbert T, Rezáčová M, Zelezná B \& Maletínská L 2011 Characterization of prolactin-releasing peptide: binding, signaling and hormone secretion in rodent pituitary cell lines endogenously expressing its receptor. Peptides 32 811-817. (doi:10.1016/j.peptides.2010.12.011)

Maletínská L, Matyšková R, MaixnerováJ, Sýkora D, Pýchová M, Spolcová A, Blechová M, DrápalováJ, LacinováZ, Haluzík M, et al. 2011 The peptidic GHS-R antagonist [D-Lys(3)]GHRP-6 markedly improves adiposity and related metabolic abnormalities in a mouse model of postmenopausal obesity. Molecular and Cellular Endocrinology 343 55-62. (doi:10.1016/j.mce.2011.06.006)

Maletínská L, Pýchová M, Holubová M, Blechová M, DemianováZ, Elbert T \& Železná B 2012 Characterization of new stable ghrelin analogs with prolonged orexigenic potency. Journal of Pharmacology and Experimental Therapeutics 340 781-786. (doi:10.1124/jpet.111.185371)

Maletinska L, Nagelova V, Ticha A, Zemenova J, PirnikZ, Holubova M, Spolcova A, Mikulaskova B, Blechova M, Sykora D, et al. 2015 Novel lipidized analogs of prolactin-releasing peptide have prolonged half-lives and exert anti-obesity effects after peripheral administration. International Journal of Obesity 39 986-993. (doi:10.1038/ijo.2015.28)

Manning S, Pucci A \& FinerN 2014 Pharmacotherapy for obesity: novel agents and paradigms. Therapeutic Advances in Chronic Disease 5 135-148. (doi:10.1177/2040622314522848)

Onaka T, Takayanagi Y \& Leng G 2010 Metabolic and stress-related roles of prolactin-releasing peptide. Trends in Endocrinology \& Metabolism 21 287-293. (doi:10.1016/j.tem.2010.01.005)

PatelD 2015 Pharmacotherapy for the management of obesity. Metabolism 64 1376-1385. (doi:10.1016/j.metabol.2015.08.001)

Phillips MS, Liu Q, Hammond HA, Dugan V, Hey PJ, Caskey CJ \& HessJF 1996 Leptin receptor missense mutation in the fatty Zucker rat. Nature Genetics 13 18-19. (doi:10.1038/ng0596-18)

Raun K, von Voss P, Gotfredsen CF, Golozoubova V, Rolin B \& Knudsen LB 2007 Liraglutide, a long-acting glucagon-like peptide-1 analog, reduces body weight and food intake in obese candy-fed rats, whereas a dipeptidyl peptidase-IV inhibitor, vildagliptin, does not. Diabetes $\mathbf{5 6}$ 8-15. (doi:10.2337/db06-0565)

Reidelberger RD, Haver AC, Apenteng BA, Anders KL \& Steenson SM 2011 Effects of exendin-4 alone and with peptide YY(3-36) on food intake and body weight in diet-induced obese rats. Obesity 19 121-127. (doi:10.1038/oby.2010.136)

Rodgers RJ, Tschöp MH \& WildingJP 2012 Anti-obesity drugs: past, present and future. Disease Models \& Mechanisms 5 621-626. (doi:10.1242/dmm.009621)

Simmons RK, Alberti KG, GaleEA, ColagiuriS, TuomilehtoJ, Qiao Q, Ramachandran A, Tajima N, Brajkovich MirchovI, Ben-Nakhi A, et al. 2010 The metabolic syndrome: useful concept or clinical tool?
Report of a WHO Expert Consultation. Diabetologia 53 600-605. (doi:10.1007/s00125-009-1620-4)

SkarbalieneJ, SecherT, JelsingJ, Ansarullah, Neerup TS, Billestrup N \& Fosgerau K 2015 The anti-diabetic effects of GLP-1-gastrin dual agonist ZP3022 in ZDF rats. Peptides 69 47-55. (doi:10.1016/j. peptides.2015.03.024)

Sturis J, Gotfredsen CF, RømerJ, Rolin B, RibelU, Brand CL, Wilken M, Wassermann K, Deacon CF, Carr RD, et al. 2003 GLP-1 derivative liraglutide in rats with beta-cell deficiencies: influence of metabolic state on beta-cell mass dynamics. British Journal of Pharmacology 140 123-132. (doi:10.1038/sj.bjp.0705397)

Sun B, Fujiwara K, AdachiS \& Inoue K 2005 Physiological roles of prolactin-releasing peptide. Regulatory Peptides 126 27-33. (doi:10.1016/j.regpep.2004.08.008)

Takayanagi Y, Matsumoto H, Nakata M, Mera T, FukusumiS, HinumaS, Ueta Y, Yada T, Leng G \& Onaka T 2008 Endogenous prolactinreleasing peptide regulates food intake in rodents. Journal of Clinical Investigation 118 4014-4024. (doi:10.1172/JCI34682)

Tatarkiewicz K, Hargrove DM, Jodka CM, Gedulin BR, Smith PA, Hoyt JA, Lwin A, Collins L, Mamedova L, Levy OE, et al. 2014 A novel long-acting glucose-dependent insulinotropic peptide analogue: enhanced efficacy in normal and diabetic rodents. Diabetes Obesity and Metabolism 16 75-85. (doi:10.1111/dom.12181)

Vaneckova I, Maletinska L, BehuliakM, Nagelova V, Zicha J \& Kunes J 2014 Obesity-related hypertension: possible pathophysiological mechanisms. Journal of Endocrinology 223 R63-R78. (doi:10.1530/JOE14-0368)

Vickers SP, Jackson HC \& Cheetham SC 2011 The utility of animal models to evaluate novel anti-obesity agents. British Journal of Pharmacology 164 1248-1262. (doi:10.1111/ j.1476-5381.2011.01245.x)

Vrang N, JelsingJ, Simonsen L, Jensen AE, Thorup I, Soeborg H \& Knudsen LB 2012 The effects of 13 wk of liraglutide treatment on endocrine and exocrine pancreas in male and female ZDF rats: a quantitative and qualitative analysis revealing no evidence of drug-induced pancreatitis. American Journal of Physiology: Endocrinology and Metabolism 303 E253-E264. (doi:10.1152/ajpendo.00182.2012)

Willms B, Werner J, Holst JJ, Orskov C, Creutzfeldt W \& Nauck MA 1996 Gastric emptying, glucose responses, and insulin secretion after a liquid test meal: effects of exogenous glucagon-like peptide-1 (GLP-1)-(7-36) amide in type 2 (noninsulin-dependent) diabetic patients. Journal of Clinical Endocrinology \& Metabolism 81 327-332.

Woods SC, Seeley RJ, Rushing PA, D'Alessio D \& Tso P 2003 A controlled high-fat diet induces an obese syndrome in rats. Journal of Nutrition 133 1081-1087.

Xiao X \& Song BL 2013 SREBP: a novel therapeutic target. Acta Biochimica et Biophysica Sinica 45 2-10. (doi:10.1093/abbs/gms112)

Yamada T, Mochiduki A, Sugimoto Y, Suzuki Y, Itoi K \& Inoue K 2009 Prolactin-releasing peptide regulates the cardiovascular system via corticotrophin-releasing hormone. Journal of Neuroendocrinology 21 586-593. (doi:10.1111/j.1365-2826.2009.01875.x)

YanovskiSZ \& YanovskiJA 2014 Long-term drug treatment for obesity: a systematic and clinical review. JAMA 311 74-86. (doi:10.1001/ jama.2013.281361)

Received in final form 19 February 2016

Accepted 23 February 2016

Accepted Preprint published online 23 February 2016 http://joe.endocrinology-journals.org

DOI: $10.1530 / J O E-15-0519$
(C) 2016 Society for Endocrinology Printed in Great Britain 